\title{
Alter und neuer Pluralismus in der Medizin
}

\author{
Robert Jütte \\ Institut für Geschichte der Medizin der Robert Bosch Stiftung, Stuttgart, Deutschland
}

\section{Schlüsselwörter}

Pluralismus in der Medizin · Komplementärmedizin .

Medizingeschichte $\cdot$ Schweiz

\section{Zusammenfassung}

Als Folge des Professionalisierungsprozesses in der Medizin seit der Mitte des 19. Jahrhunderts wurde eine Reihe von neuen Heilweisen wie Homöopathie, Wasserheilkunde oder Mesmerismus als «Quacksalberei» eingestuft. Als sich der Terminus Komplementärmedizin und Alternativmedizin (CAM) im letzten Drittel des 20. Jahrhunderts durchsetzte, änderte sich die Bedeutung des Begriffs «Medizinischer Pluralismus», indem der Kurpfuscher nicht mehr derjenige war, der eine unkonventionelle medizinische Methode vertrat. Die neue Grenzziehung erfolgte aufgrund ethischer Kriterien, wie z.B. Kompetenz, Qualifikation, Verhalten, Verantwortung und Professionalität, unabhängig von der Therapieform. Diese Entwicklung wird auch unter dem Begriff «neuer Pluralismus» gefasst. Die Schweiz gehört zu den ersten Staaten in Europa, in denen die direkte Demokratie über den Volksentscheid das Gesundheitssystem zwang, CAM-Verfahren offiziell anzuerkennen.

\section{Keywords}

Medical pluralism $\cdot$ Complementary medicine $\cdot$ Medical history Switzerland

\section{Summary}

Old and New Medical Pluralism

Along with the professionalization of medicine in the mid-19th century a wide range of new medical movements such as homeopathy, hydropathy, or mesmerism were categorized as 'quackery'. In the late 20th century, the birth of 'complementary and alternative medicine' (CAM) resulted in yet another transformation into 'medical pluralism', defining quackery no longer as adhering to an unconventional treatment. The line of demarcation can now be found in a more ethical field, e.g. competency, qualifications, conduct, responsibility, and personal professional development of a practitioner, almost regardless of the form of therapy in question. This development has been labelled 'new pluralism'. Switzerland is among the first countries in Europe where direct democracy via referendum paved the way to a medical health care system recognising CAM treatments.

\section{Mots-clés}

Pluralisme médical · Médecine complémentaire .

Histoire de la médecine · Suisse

\section{Résumé}

Ancien et nouveau pluralisme médical

A la suite du processus de professionalisation de la médecine qui s'est entrepris dès la seconde moitié du $19^{e}$ siècle, de nombreux nouveaux modes de guérison, tels que I'homéothérapie, I'hydrothérapie ou encore le mesmérisme, se sont vus qualifiés de «charlatanisme». Tandis que le terme de Médecine alternative complémentaire (MAC) s'est imposé dans le dernier tiers du $20^{\mathrm{e}}$ siècle, le concept de «pluralisme médical» a changé de signification: le représentant d'une méthode de médecine non conventionnelle n'était plus perçu de façon systématique comme un charlatan. Cette nouvelle distinction s'est opérée en se basant sur des critères éthiques, tels que la compétence, la qualification, le processus, la responsabilité et le professionnalisme, indépendamment de la forme de traitement. On utilise également le terme «pluralisme nouveau» pour décrire cette évolution. La Suisse fait partie des premiers pays en Europe où la démocratie directe, par le biais d'un référendum, a obligé le système de santé à reconnaître officiellement les procédés de MAC.

\section{Begrifflichkeiten}

Mitte der 1920er Jahre fand sich im deutschsprachigen Raum eine Gruppe von Ärzten zusammen, die von der Notwendigkeit überzeugt war, die verschiedenen therapeutischen Richtungen «im Sinne einer naturwissenschaftlich begründeten und zur Kunst entwickelten Heilkunde» [1] zu vereinigen. Ihr Motto war: «Ars una, species

\section{KARGER \\ Fax +497614520714 Information@Karger.d} www.karger.com
Prof. Dr. phil. Robert Jütte

Institut für Geschichte der Medizin der Robert Bosch Stiftung

Straussweg 17, 70184 Stuttgart, Deutschland

Tel. +49 711 46084-171, Fax -181

robert.juette@igm-bosch.de 


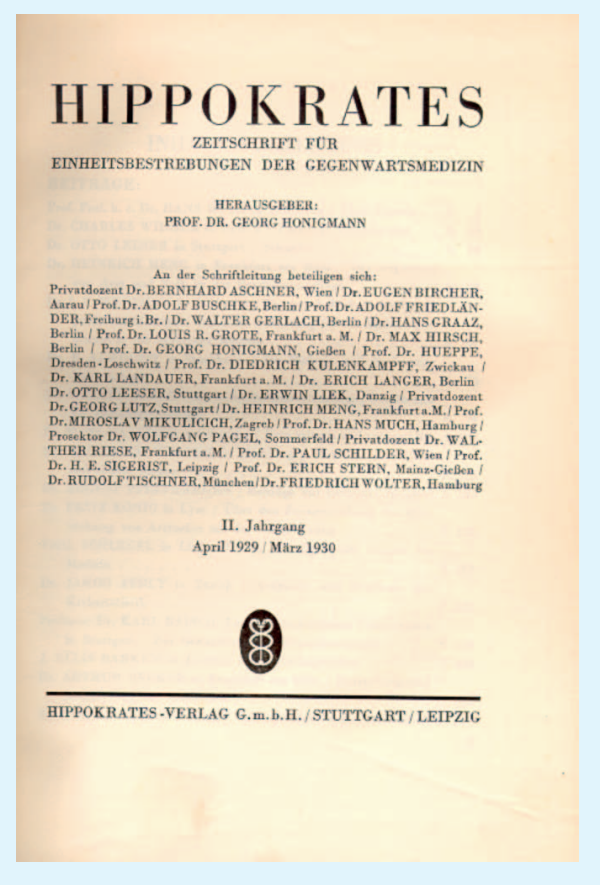

Abb. 1. Zeitschrift Hippokrates.

mille» (Eine Kunst, tausend Unterarten). Das Sprachrohr bzw. die publizistische Plattform dieser Bewegung war die Zeitschrift Hippokrates, die 1929 begründet wurde (Abb. 1). Ihr programmatischer Untertitel lautete: «Zeitschrift für Einheitsbestrebungen der Gegenwartsmedizin». Der Herausgeber, Georg Honigmann (1863-1930), machte in der ersten Nummer dieser Zeitschrift die Zielsetzung deutlich [2]:

\begin{abstract}
«Die Medizin darf in ihrer Entwicklung nicht an das letzten Endes zufällige, ihr durchaus nicht adäquate Neben- und Nacheinander naturwissenschaftlicher Entdeckungen allein gebunden sein, wenn sie auch der Befruchtung durch sie keineswegs entraten soll. Sie muss von einer einheitlichen Entwicklungsidee geleitet und durchwaltet werden, in deren Aufbau sie dazu gelangt, den Sinn der menschlichen Krankheitserscheinungen zu verstehen.»
\end{abstract}

Diese Reformbestrebungen der 1920er Jahre müssen vor dem Hintergrund einer Debatte gesehen werden, die damals in Deutschland mit dem Schlagwort «Kurierfreiheit» geführt wurde (Abb. 2). Heute würden wir in diesem $\mathrm{Zu}$ sammenhang vom «Medizinischen Pluralismus» sprechen.

Der Begriff «Medizinischer Pluralismus» ist jüngeren Datums. Er stammt aus der Medizinischen Anthropologie bzw. Medizinsoziologie [3-5]. Es gibt unterschiedliche theoretische Ansätze. So hebt z.B. Charles Leslie auf die kulturelle und soziale Dimension unterschiedlicher Medizinformen ab [6]. Medizinsysteme können demnach sowohl aus biologischer, kultureller und sozialer Perspektive betrachtet werden. In jedem Medizinsystem lassen sich seiner Ansicht nach pluralistische Strukturen unterschiedlicher Typen von Praktikern und institutionellen Normen feststellen. Von einem pluralistischen Medizinsystem mit Interaktionen zwischen medizinischen Sub-

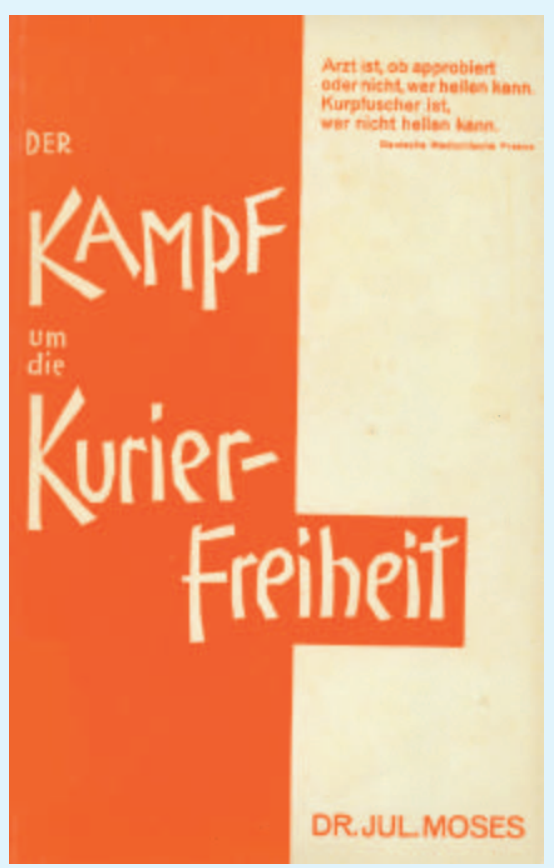

Abb. 2. Die Debatte «Der Kampf um die Kurierfreiheit».

systemen geht dagegen der amerikanische Medizinethnologe Arthur Kleinman aus. Statt «Medizinsystem» bevorzugt er jedoch den Begriff «health care system». Nach seiner Auffassung schliesst dieser Terminus sowohl die Prävention als auch die Heilfunktion ein [7]. Kleinman unterscheidet bei den Faktoren, die den Medizinbereich beeinflussen, äussere und innere. Beide üben einen Einfluss auf das Medizinsystem aus. Für strukturelle Veränderungen im Gesundheitssystem sind z.B. Politik, Wirtschaft, soziale Schichtung und technische Entwicklungen verantwortlich. Die Innenstruktur des jeweiligen Gesundheitssystems besteht nach Kleinman aus folgenden drei Sektoren: 1) der professionellen Medizin, 2) der populären Medizin, die von Laienheilern angeboten wird, und 3) der Volksmedizin, die vor allem von Familienangehörigen praktiziert wird (Stichwort «Hausmittel») [8]. Keiner dieser drei Sektoren existiert unabhängig von den anderen. Sie überlappen sich, wenn der Kranke - was nicht selten vorkommt - entweder nacheinander oder gleichzeitig medizinische Hilfe seitens seiner Familie, eines Arztes oder eines Wunderheilers in Anspruch nimmt. Dabei kann er entweder in die Rolle des kranken Familienangehörigen, Patienten oder Klienten schlüpfen.

Die «Wiederentstehung» (engl. re-emergence) und zunehmende Legitimierung der Alternativen Medizin, die seit den 1980er Jahren in den USA und in Westeuropa zu beobachten ist [9], hat zwei englische Forscherinnen, Sarah Cant und Ursula Sharma, dazu geführt, von einem «neuen medizinischen Pluralismus» zu sprechen [10]. Die Anwendung alternativer therapeutischer Systeme wird von ihnen als eine Herausforderung für die biomedizinische Autorität gesehen. Verschiedene Faktoren sollen eine in- 
zwischen positivere Haltung des Staates zur Alternativen Medizin begünstigt haben, darunter nicht zuletzt gesundheitsökonomische Überlegungen. Gleichzeitig sei eine Neugestaltung des Expertenwissens («refiguring of expertise») zu beobachten. Der moderne Staat sei nämlich auf die Sachkenntnis der verschiedenen Professionen angewiesen. Die Anwender alternativer Therapien hätten vor allem von Verschiebungen in den sozialen Beziehungen zwischen Biomedizin, Regierung und Verbraucher profitiert. Da sich diese Form des Pluralismus, wie er sich im späten 20. Jahrhundert entwickelte, von dem der «vormodernen» Zeit angeblich erheblich unterscheidet, haben Cant und Sharma ihn als «neuen» medizinischen Pluralismus bezeichnet [11].

\section{Ein Blick in die Geschichte}

Von einem medizinischen Pluralismus im heutigen Sinne des Wortes kann also vor Ende des 19. Jahrhunderts keine Rede sein, da es noch keine «Schulmedizin» oder wie es heute im Sozialgesetzbuch der Bundesrepublik Deutschland heisst - einen «allgemein anerkannten Stand der medizinischen Erkenntnisse» ( $\$ 2$, Absatz 1) gab [12]. Ganz im Gegenteil: Das Lager der gelehrten Ärzte war heillos zerstritten [13]. So hielt man in der Krankheitslehre weitgehend an der seit der Antike wirkmächtigen Lehre von den vier Körpersäften fest, zwängte aber Erscheinungen, die man am gesunden oder kranken Menschen beobachtete, in verschiedene Systeme, die wie Moden auftraten und wieder in der Versenkung verschwanden. Noch um die Wende vom 18. zum 19. Jahrhundert waren die medizinischen Lehrbücher so buntscheckig wie die Landkarte des Heiligen Römischen Reiches Deutscher Nation. Entsprechend vielfältig war das Therapieangebot der gelehrten Ärzte. Neben dem therapeutischen Dreigestirn (Aderlass, Brech- und Abführmittel), das die sogenannte «heroische Medizin» dieser Zeit kennzeichnete, gab es, wie ein Blick in ein Standardwerk der Medizin um 1800 zeigt, noch "specifische», "resolvirende», «anti-miasmatische», «excitirende» und andere Methoden, mit denen Ärzte ihre duldsamen Patienten zu kurieren versuchten [14].

Der Erfolg galt keinesfalls als das einzige und allgemeingültige Beurteilungskriterium für ärztliches Handeln. War es in jener Zeit noch die «ars medicina», die Heilkunst, die als Rechtfertigung für die eigene Überlegenheit diente, so übernahm im 19. Jahrhundert die Wissenschaft, genauer gesagt die Naturwissenschaft, diese Rolle. Dennoch blieb die handwerklich-zünftige Tradition des offiziellen Medizinalwesens, insbesondere im Umgang mit den sogenannten «Kurpfuschern» $[15,16]$, auch im 19. Jahrhundert «unter veränderten sozialpolitischen Bedingungen, für die Organisation, Artikulation und Durchsetzung berufsständischer Interessen der Ärzte und der sich professionalisierenden Schulmedizin von Bedeutung» [17].

Seit der zweiten Hälfte des 19. Jahrhunderts war die Homöopathie, der ein Leipziger Gericht 1830 bescheinigt hatte, ein medizinisches System wie jedes andere zu sein und damit staatlicherseits nicht verboten werde könne, längst nicht mehr der Hauptgegner der sich hinter dem Banner der «wissenschaftlichen Medizin» versammelnden Ärzteschaft [9]. Man fühlte sich sehr viel stärker von den «Naturärzten» bedroht, die oft keine approbierten Mediziner, sondern heilkundige Laien waren. Es kam hinzu, dass seit 1873 reichsweit die Gewerbeordnung des Norddeutschen Bundes galt, welche die Kurierfreiheit, d.h. die fast uneingeschränkte Ausübung der Heilkunde durch praktisch jedermann, gesetzlich verankert hatte. Während im Regierungsentwurf von 1869 noch vorgesehen war, die Berechtigung zur Ausübung der Heilkunde an einen Befähigungsausweis (Approbation) zu binden, plädierte die einflussreiche Berliner Medizinische Gesellschaft, der solche Ärztepersönlichkeiten wie Rudolf Virchow und August Hirsch angehörten, für die Gewerbe- und damit für die Kurierfreiheit. Nach einer heftigen Aussprache billigte der Reichstag des Norddeutschen Bundes am 25. Mai 1869 die Neufassung des Paragraphen 29 der Gewerbeordnung. Die Kurierfreiheit wurde damit zunächst im Bundesgebiet eingeführt, und die alten medizinalgesetzlichen Bestimmungen wurden ausser Kraft gesetzt. Bis 1873 hatten alle Bundesstaaten des Deutschen Reiches die Gewerbeordnung übernommen. Damit war es jedermann gestattet, die Heilkunde in all ihren Sparten auszuüben. Nur durfte sich niemand als «Arzt» bezeichnen oder sich ähnlich lautende Titel zulegen, der nicht approbiert war oder eine entsprechende staatliche Erlaubnis besass. Diese Liberalisierung des Gesundheitsmarktes, die vor mehr als 130 Jahren eingeleitet wurde, prägt trotz aller Versuche der Einschränkung bis heute den medizinischen Pluralismus und erklärt, dass es in keinem anderen Land der Welt den Berufsstand der Heilpraktiker gibt [18].

Zwar gelang es der organisierten Ärzteschaft im Kaiserreich nicht, die Gewerbeordnung durch eine Ärzteordnung in ihrem Sinne zu ersetzen, doch erreichte man immerhin einige kleine Abänderungen. So durften Heilkundige ihr Gewerbe seit 1883 nicht mehr im Umherziehen ausüben. Bereits einige Jahre zuvor hatte man den Betrieb von Privatkrankenanstalten und Badeanstalten von einer Konzession abhängig gemacht. Ansonsten blieb den Ärzten immer noch die Möglichkeit, strikt auf die vorhandenen gesetzlichen Bestimmungen für die Ausübung des Heilgewerbes zu achten und das Tätigkeitsfeld der lästigen Konkurrenten durch entsprechende Erlasse und rechtsbildende Entscheidungen einzelner Gerichte einzuschränken (Abb. 3). Vor allem verfolgte man seitens der ärztlichen Standesorganisationen mithilfe des bestehenden Strafrechts diejenigen Heiler, die sich einen 


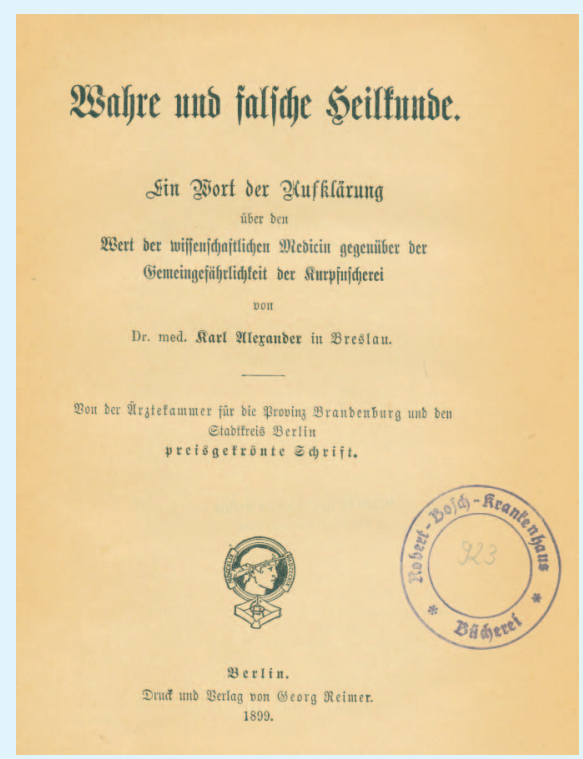

Abb. 3. Veröffentlichung «Wahre und falsche Heilkunde» aus dem Jahr 1899.

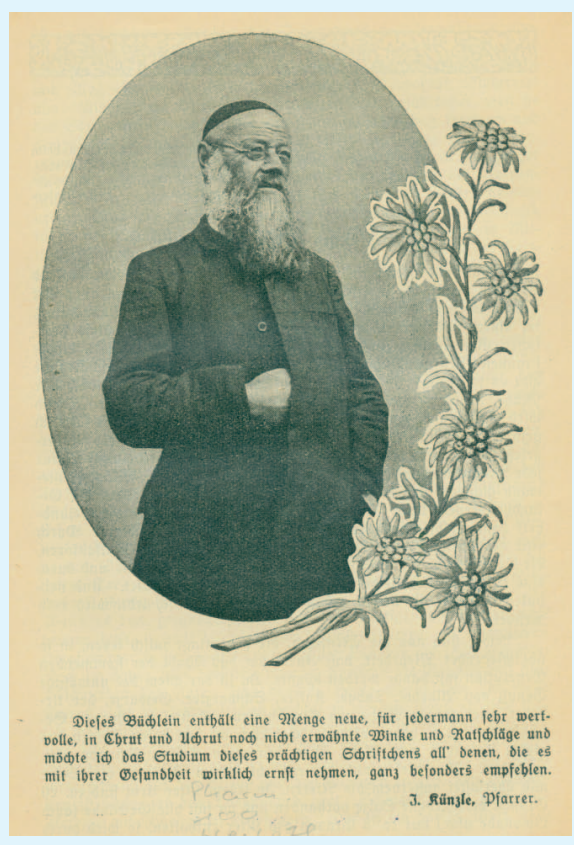

Abb. 4. «Chrut und Uchrut» des Schweizer Pfarrers und Naturheilkundigen Johann Künzle (1857-1945).

arztähnlichen Titel («Homöopath», «Magnetopath», «Naturarzt» oder "geprüfter Naturheilkundiger») zulegten. Noch um 1905 durften sich selbst approbierte Mediziner nicht «Arzt für Naturheilverfahren» nennen, wenn sie nicht mit der Standesgerichtsbarkeit in Konflikt kommen wollten [19]. Ausserdem berief man sich von ärztlicher Seite häufig auf die einschlägigen Bestimmungen des «Reichsgesetzes zur Bekämpfung des unlauteren Wettbewerbs» von 1896.

Neben der organisierten Ärzteschaft war es vor allem die «Deutsche Gesellschaft zur Bekämpfung der Kurpfuscherei», die gegen angebliche Missstände im Heilgewerbe ankämpfte und eine Vielfalt von Aktivitäten entfaltete. In der von ihr herausgegebenen Zeitschrift Der Gesundheitslehrer erschien 1932 ein Artikel mit dem Titel
«Moral und Recht im Kurpfuschertum». Darin wurde gefordert, jedem, der wirklich Kenntnisse und Fähigkeiten auf dem Gebiet der Heilkunde besitzt, zu ermöglichen, eine staatliche Genehmigung zur Ausübung dieser Heilkunde zu erlangen. Die hier geforderte «kleine Approbation» sollte die Gewähr dafür bieten, dass jeder Heilkundige "eine moralisch einwandfreie Persönlichkeit» und «kein Pfuscher» sei [20]. Hier deutet sich bereits die Lösungsmöglichkeit an, die 1939 schliesslich unter geänderten politischen Bedingungen Eingang in das «Gesetz über die berufsmässige Ausübung der Heilkunde ohne Bestallung (Heilpraktikergesetz)» fand. Der sich in der Endphase der Weimarer Republik anbahnende Kompromiss trug zweifellos mit dazu bei, dass die KurpfuscherDebatte, die über viele Jahrzehnte die Gemüter bewegt hatte, allmählich abebbte und nur noch gelegentlich wieder aufflackerte.

\section{Der Blick über den Zaun: Medizinischer Pluralismus in der Schweiz - damals und heute}

Im Kanton Graubünden forderte 1921 eine Volksinitiative die Freigabe der «giftfreien Kräutermethode», die damals von dem in Zizers lebenden Schweizer Pfarrer und Naturheilkundigen Johann Künzle (1857-1945) ausging [21]. Dieser war der Verfasser eines populärmedizinischen Ratgebers, der den schönen schwyzerdütschen Titel «Chrut und Uchrut» trägt (Abb. 4). Schon während seines Theologiestudiums beschäftigte Künzle sich mit der Botanik und der Heilkraft der Pflanzen [9]. In den abgelegenen Schweizer Bergorten, in denen er später seinen Dienst als Pfarrer versah, hatte er reichlich Gelegenheit, seine Heilkräuterkenntnisse an der bäuerlichen Bevölkerung zu erproben. Bald konnte er sich vor Hilfe suchenden Patienten nicht mehr retten, gab sein Amt als Seelsorger auf und widmete sich fortan ganz der Kräuterheilkunde. Anfang der 1920er Jahre war der Name «Kräuterpfarrer Künzle» bereits weit über die Schweizer Landesgrenzen hinaus gedrungen. Sein Kräuterbuch «Chrut und Uchrut» wurde rasch in mehrere Sprachen übersetzt. Bald schon mussten die Produkte (Kräutertees, Elixiere usw.), die seinen $\mathrm{Na}$ men trugen, gesetzlich gegen Missbrauch geschützt werden. Zur Vermarktung dieser Produkte trug nicht zuletzt eine eigene Monatsschrift bei, die den Namen Salvia trug und den Lesern Rat versprach, wie «manche Krankheiten auf einfache und wirksame Weise zu heilen sind» [9].

Die Nachfrage nach solch kräuterheilkundlicher Literatur war offenbar so gross, dass sie von Künzle allein nicht befriedigt werden konnte. So erschien bereits 1917 ein «Nachtrag» zu Pfarrer Johann Künzles Heilkräuterbüchlein aus der Feder eines gewissen J. Gyr-Niederer aus Gais im Kanton Appenzell, von dem bis 1926 über 200000 Exemplare verkauft wurden. In einer der vielen Auflagen 
dieser ebenfalls weit verbreiteten Schrift wird das Selbstverständnis eines überzeugten Kräuterarztes und Laienheilers deutlich [22]:

\begin{abstract}
«Wenn nun die Herren Aerzte ihr grosses Wissen dazu benützen würden, in Zukunft weniger giftige, für den Körper schädliche Mittel, dafür mehr giftfreie, natürliche Anwendungen zu verordnen, sowie noch mehr für Krankheitsvorbeugung einzutreten, dann kann und wird der Laie seine Siebensachen zusammenpacken und den staatlichen Vertretern der medizinischen Wissenschaft das Arbeitsfeld der hehren Heilkunst kampflos überlassen.»
\end{abstract}

Von einer Anerkennung der Kräutermedizin oder auch nur einer Tolerierung durch die Schulmedizin war man, wie dieses Zitat zeigt, zumindest in den ersten beiden Jahrzehnten des 20. Jahrhunderts noch weit entfernt. Dennoch gab es Anfang der 1920er Jahre zumindest einen Teilsieg für diese alternative Heilweise. Vorausgegangen war eine Pressekampagne von Gegnern und Befürwortern dieses Aussenseiterverfahrens [23]. Die einen verwiesen darauf, dass der Patient, der in der Schulmedizin keine Heilung findet, das Recht habe, diese beim «Naturarzt» zu suchen. Dagegen argumentierte z.B. der Bündnerische Ärzteverein, dass man niemanden heilen könne, «sei es mit oder ohne Kräuter (...), ohne erst die Krankheit zu kennen» (zitiert in [21]). Die Volksabstimmung fand am 30. April 1922 statt. Was die einen sich erhofft und die anderen befürchtet hatten, trat ein: Die grosse Mehrheit der Graubündener (12 602 Ja-Stimmen gegen 8428 Nein-Stimmen) sprach sich für die Zulassung der Kräuterheilkunde nach Pfarrer Künzle aus. Doch die Bedingung staatlicherseits war, dass sich der populäre Naturheilkundige einer Prüfungskommission stellte. Das Examen unter der Beteiligung eines Schulmediziners fand am 10. Juli 1922 im Regierungsgebäude in Chur statt. Pfarrer Künzles Antworten auf medizinische Fragen wurden «als populäre(r) Darstellung genügend» befunden und ihm die Ausübung der Heilkunde bewilligt. Auch sein Nachfolger, Albert Emmenegger (1857-1945) in Maienfeld, erhielt 1940 nach einer Prüfung, die ebenfalls nicht einfach zustande kam, die Zulassung als Heiler [24]. Heute wirbt eine Schweizer Internetseite mit folgendem vollmundigen Hinweis für die von Pfarrer Künzle propagierte Kräuterheilkunde [25]:

\footnotetext{
«Als Kräuter-Pfarrer Künzle auch Zustimmung von Kaiser FranzJosef erhielt, fand er sogar Anerkennung bei jenem Ärztekollegium, das seine Arbeit verbieten wollte. Mit seinem enormen medizinischen Wissen und seiner herausragenden Intelligenz (8 Sprachen) konnte er schliesslich alle davon überzeugen, dass viele Gesundheitsprobleme mit seinen pflanzlichen Präparaten erfolgreich behandelt werden können. Er gilt somit als der Wegbereiter der modernen Phyto-Therapie (Pflanzenheilkunde) und war ein Vorreiter der Ganzheitsmedizin ... und heute sind seine phyto-therapeutischen (pflanzlichen) Produkte aktueller denn je.»
}

Anfang der 1930er Jahre musste die Schweizer Bevölkerung erneut darüber entscheiden, ob eine damals als Aussenseiterverfahren bezeichnete Heilmethode offiziell zugelassen werden sollte oder nicht. Zu dieser Zeit gab es in Luzern und Zürich drei Chiropraktoren, die immer wieder mit den Medizinalgesetzen in Konflikt gerieten, was ihrer Popularität allerdings keinen Abbruch tat. So erteilte ein Luzerner Gericht dem Chiropraktor Josef Theodor Müller ein Praxisverbot [26]. Doch dieser gab den Kampf um Anerkennung nicht auf und mobilisierte mit dem 1930 von ihm gegründeten «Verein zur gesetzlichen Anerkennung der Chiropractic» zahlreiche Menschen. Diese Bürgerinitiative erreichte schliesslich 1934 eine Begnadigung Müllers. Doch man ging noch weiter und wollte eine gesetzliche Anerkennung der Chiropraktik. Ein «Initiativbegehren für die Zulassung der chiropractischen Heilmethode» wurde am 10. März 1934 bei der Staatskanzlei angemeldet. Bis zum Sommer hatte man ca. 17000 Unterschriften zusammen. Im November 1935 wandte sich die Luzerner Sektion des Vereins zur gesetzlichen Anerkennung der Chiropractic in der Schweiz wie folgt an den Grossen Rat des Kantons Luzern [26]:

\begin{abstract}
«Mit der Einreichung des Volksbegehrens für die gesetzliche Anerkennung der Chiropractic ist eindeutig zum Ausdruck gekommen, dass weit und breit diesem Heilverfahren ein breites Interesse entgegengebracht wird. (...) Was wir wollen und erstreben ist keine schrankenlose Freiheit und Zügellosigkeit. Wir wollen einzig und allein, dass innerhalb genau umschriebener gesetzlicher Bestimmungen für die Chiropractic die Möglichkeit der Hilfe gegenüber der leidenden Menschheit gewährt werde.»
\end{abstract}

Am 25. Februar 1937 beschloss der Grosse Rat mit Mehrheit, dem Volksbegehren zu entsprechen und die Chiropraktik als Heilkunde offiziell zuzulassen.

Im Juni 1936 reichte auch der Zürcher Verein zur gesetzlichen Anerkennung der Chiropraktik in der Schweiz ein entsprechendes Initiativbegehren ein (Abb. 5). Daraufhin setzte die Gesundheitsdirektion eine Expertenkommission ein, um die Bedeutung der Chiro-

Abb. 5. Initiativbegehren zur gesetzlichen Anerkennung der Chiropraktik in der Schweiz (1936).
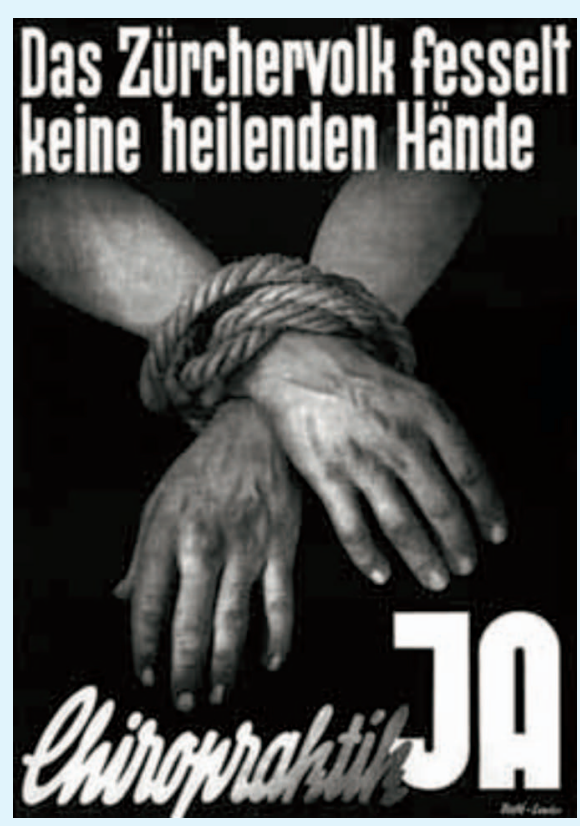
praktik «gründlich und unvoreingenommen» abzuklären. Die Kommission, in der zahlreiche namhafte Schulmediziner vertreten waren, kam in einem 199 Seiten umfassenden Gutachten zu dem Schluss, die Chiropraktik sei die willkürliche und unbewiesene Theorie eines amerikanischen Spezereihändlers. Sie werde in Amerika an sogenannten Hochschulen gelehrt, deren Programm nicht viel über dem einer schweizerischen Sekundarschule stehe. Die angeblichen Heilerfolge dieser Theorie hielten einer wissenschaftlichen Überprüfung nicht stand [27]. Dieses negative Urteil veranlasste den Regierungs- wie auch den Kantonsrat, das Begehren abzulehnen. Die Stimmbürger entschieden aber wider Erwarten anders. Ein Volksentscheid im Januar 1939 erbrachte eine klare Mehrheit für das Initiativbegehren. Der Ausgang der Volksabstimmung sollte gesamtschweizerisch Schule machen. So lehnten sich beispielsweise sämtliche Universitätskantone an das Vorbild von Zürich an. In Basel-Stadt wurde auch die Ausbildung gesetzlich geregelt, indem festgelegt wurde, dass unter chiropraktischer Tätigkeit das zu verstehen sei, was an den Chiropraktik-lnstituten gelehrt und gelernt und von den Prüfungen in der Schweiz examiniert werde. Nach dem erfolgreichen Kampf um den Pluralismus in der Medizin, der zunächst auf Kantonsebene geführt wurde, gelang es schliesslich mit massiver Unterstützung aus der Bevölkerung (eine Unterschriftenaktion, an der sich über 400000 Schweizer beteiligten), die chiropraktische Tätigkeit in den Leistungsbereich zunächst der Kranken- und später auch in die Unfall- bzw. Militärversicherung einzubeziehen, und zwar im Rahmen der Revision des Kranken- und Unfallversicherungsgesetzes (KUVG) im Jahre 1963/1964. Der rechtliche Status des Chiropraktors ist heute sowohl in den kantonalen Gesundheitsgesetzen als auch in der Bundesgesetzgebung über die Sozialversicherung geregelt. Der Luzerner Verein «Pro Chiropraktik» (LVPC) besteht übrigens bis heute und legt Zeugnis darüber ab, wie früh schon Patienten für den Pluralismus in der Medizin eintraten und wie schwierig, aber letztendlich doch erfolgreich dieser Kampf war.

Auch in jüngster Zeit war es wieder ein Bürgerbegehren, das die Einbeziehung alternativer Verfahren in den Leistungskatalog der Grundversicherung forderte. Die Volksinitiative «Ja zur Komplementärmedizin» wurde 2005 ge- gründet und sammelte in kürzester Zeit knapp 139000 Unterschriften. Im August 2006 beantragte der Bundesrat, das Volksbegehren ohne Gegenvorschlag zu verwerfen. Ein Jahr zuvor hatte Gesundheitsminister Couchepin fünf komplementäre Behandlungsmethoden - Homöopathie, Neuraltherapie, Phytotherapie sowie die Angebote der chinesischen und Anthroposophischen Medizin - aus dem Katalog der Grundversicherung streichen lassen und damit den offenen Widerstand der in der Schweiz tätigen alternativen Therapeuten, aber auch der Patienten herausgefordert. Umfragen zeigten, dass die Mehrheit der Bevölkerung Komplementärmedizin wünschte [28]. So ergab eine Umfrage des Krankenkassenverbandes santésuisse, dass sich $82 \%$ der Bevölkerung für eine umfassende Berücksichtigung der Komplementärmedizin aussprachen. So fiel das Ergebnis der Volksabstimmung am 17. Mai 2009 klar aus. Über zwei Drittel der abgegebenen Stimmen (67\%) waren für den Vorschlag der Volksinitiative «Ja zur Komplementärmedizin». Die neue Verfassungsbestimmung, die auf diese Weise mehrheitlich Zustimmung gefunden hat, lautet: «Bund und Kantone sorgen im Rahmen ihrer $\mathrm{Zu}$ ständigkeiten für die Berücksichtigung der Komplementärmedizin.» Doch die Umsetzung gestaltet sich bis heute schwierig, da aus der vom Volk erzwungenen Gesetzesänderung nicht eindeutig hervorging, dass komplementärmedizinische Methoden Aufnahme in die obligatorische Grundversicherung finden müssen [29]. Ab dem 1. Januar 2012 wurden jedoch die fünf ärztlichen Methoden der Komplementärmedizin - Anthroposophische Medizin, Klassische Homöopathie, Störfeldtherapie (Teil der Neuraltherapie), Phytotherapie und Traditionelle Chinesische Medizin (TCM) - provisorisch für 5 Jahre wieder in den Leistungskatalog der Grundversicherung aufgenommen. Dies erfolgte nach einem Entscheid des damaligen Bundesrates Didier Burkhalter im Januar 2011. Trotz vorhandener Hindernisse wird man der Schweiz somit attestieren müssen, dass sie das einzige Land in Europa ist, in dem der Pluralismus in der Medizin gesetzlich verankert ist und eine relativ lange Tradition aufweist.

\section{Disclosure Statement}

Es bestehen keine Interessenkonflikte.

\section{Literatur}

1 Geleitwort. Hippokrates 1929;1:1-2.

2 Honigmann G: Einheitsbestrebungen der Gegenwartsmedizin. Zur Einführung. Hippokrates 1929;1:3-15.

3 Pfleiderer B, Bichmann W: Krankheit und Kultur. Eine Einführung in die Ethnomedizin. Berlin, Reimer, 1985.
4 Ernst W (Hrsg): Plural Medicine, Tradition and Modernity, 1800-2000. London, Routledge, 2002.

5 Bruchhausen W: Medizin zwischen den Welten. Geschichte und Gegenwart des medizinischen Pluralismus im südöstlichen Tansania. Bonn, Bonner Universitätspresse/V\&R unipress, 2006.
6 Leslie C (ed): Asian Medical Systems: A Comparative Study. Berkeley, University of California Press, 1976.

7 Kleinman A: Concepts and a model for the comparison of medical systems as cultural systems. Soc Sci Med 1978;12:85-93. 
8 Kleinman A: Patients and Healers in the Context of Culture: An Exploration of the Borderland between Anthropology Medicine and Psychiatry. Berkeley, University of California Press, 1980.

9 Jütte R: Geschichte der Alternativen Medizin. Von der Volksmedizin zu den unkonventionellen Therapien von heute. München, C.H. Beck, 1996.

10 Cant S, Sharma U: A New Medical Pluralism? Alternative Medicine, Doctors, Patients and the State. London, Routledge, 1999.

11 Cheng H: Medizinischer Pluralismus und Professionalisierung - Entwicklung der Chinesischen Medizin in Taiwan. Sozialwissenschaftliche Dissertation. Bielefeld, 2003. deposit.ddb.de/cgi-bin/dokserv?idn=966622065.

12 Jütte R: Pluralismus in der Medizin aus historischer Perspektive; in Michl M, Potthast T, Wiesing U (Hrsg): Pluralität in der Medizin. Werte Methoden - Theorien. Freiburg i.Br., Alber, 2008, pp 381-394.

13 Stenzel O: Medikale Differenzierung: der Konflikt zwischen akademischer Medizin und Laienheilkunde im 18. Jahrhundert. Heidelberg, Auer, 2005.

14 Hufeland CW: System der practischen Heilkunde, 2 Bände. Jena, Frommann, 1800/1802.

15 Teichler JU: «Der Charlatan strebt nicht nach Wahrheit, er verlangt nur nach Geld»: Zur Auseinandersetzung zwischen naturwissenschaftlicher Medizin und Laienmedizin im deutschen Kaiserreich am Beispiel von Hypnotismus und Heilmagnetismus. Stuttgart, Franz Steiner, 2002.

16 Winnick TA: From quackery to 'complementary' medicine: the integration of alternative therapies in the American medical profession. Dissertation. Ann Arbor/Michigan, Indiana University, 2001.

17 Braun R: Zur Professionalisierung des Ärztestandes in der Schweiz; in Conze W, Kocka J (Hrsg): Bildungsbürgertum im 19. Jahrhundert. Teil 1: Bildungssystem und Professionalisierung im internationalen Vergleich. Stuttgart, Klett-Cotta, 1985, pp 332-357.

18 Faltin T: «Heilpraktiker»; in Gerabek WE, Haage BD, Keil G, Wegner W (Hrsg): Enzyklopädie Medizingeschichte. Berlin, de Gruyter, 2005, pp 554-555.

1940 Jahre Naturheilbewegung. Festschrift zum 40-jährigen Bestehen des Deutschen Bundes für naturgemässe Lebens- und Heilweise (Naturheilkunde) e.V. Berlin, Selbstverlag, 1929.

20 Lang (Dr.): Moral und Recht im Kurpfuschertum. Der Gesundheitslehrer 1932;35:199-200.

21 Bündnerischer Ärzteverein (Hrsg): Äskulap in Graubünden. Beiträge zur Geschichte der Medizin und des Ärztestandes. Chur, Calven, 1970.

22 Gyr-Niederer J: Nachtrag zu Pfarrer Joh. Künzle’s Heilkräuterbüchlein 'Chrut und Uchrut'. Ausgabe 145 000-190 000. Gais/Appenzell, Selbstverlag, 1917.

23 Metz von Calven P: Geschichte des Kantons Graubünden, Band 3. Chur, Calven, 1993.

24 Staatsarchiv Graubünden. Bestand Bündnerischer Ärzteverein DV_10 Nr. 6.

25 Kräuter-Pfarrer Künzle. www.kp-kuenzle.ch

26 Verein «Pro Chiropractic» Sektion Luzern (Hrsg): Die Geschichte der Chiropractic im Kanton Luzern. Luzern, Selbstverlag, 1937.

27 Naef JP: 50 Jahre Schweizerische Chiropraktoren Gesellschaft SCG. www.chiropraktik.ch/Deutsch/Geschichte/50J_SCG.cfm.

28 Wolf U, Wolf M, von Ammon K: Rahmenbedingungen und Inanspruch nahme von CAM in der Schweiz; in Bornhöft G, Matthiessen PF (Hrsg) Homöopathie in der Krankenversorgung - Wirksamkeit, Nutzen, Sicherheit und Wirtschaftlichkeit. Frankfurt/M., VAS, 2006, pp 138-152.

29 Schoch C: Offizielle Akzeptanz der Komplementärmedizin. Am Beginn eines langen Wegs durch die Instanzen. Neue Zürcher Zeitung. 17.5.2009. 